\title{
Aportes de las académicas latinoamericanas para comprender las acciones colectivas de las mujeres ${ }^{* *}$
}

\author{
Contributions of Latin American female academics to \\ understand the collective actions of women
}
Contribuições das acadêmicas latino-americanas para compreender as ações coletivas das mulheres

\footnotetext{
* Socióloga, Doctora en Ciencias Sociales, profesora del Departamento de Ciencias Sociales de la Universidad del Valle, integrante del grupo Acción Colectiva y Cambio Social, ACASO. Correo electrónico: maria.ibarra@correounivalle.edu.co

** Este artículo es resultado de la investigación Activismo por la paz en Colombia. Mujeres e indígenas en el gobierno doble de la seguridad democrática (2002-2010). El proyecto fue financiado por la Vicerrectoría de Investigaciones de la Universidad del Valle, en la convocatoria 2011, y se realizó en coautoría con el profesor Jorge Hernández, del departamento de Ciencias Sociales de la misma Universidad. Artículo recibido el 18/02/2015 y aceptado el 05/04/2015.
} 


\section{Resumen}

Abstract

Resumo

Este artículo presenta un balance de la producción académica sobre la participación política de las mujeres en acciones colectivas promovidas por organizaciones sociales, redes y el movimiento de mujeres y feminista en América Latina y Colombia, publicada en el período 1990-2014. Este recuento prioriza el análisis de contenido de los textos, se centra en la forma como sus autoras construyen los objetos de investigación, en los referentes teóricos y en los aportes al conocimiento de la relación género y acción colectiva. Las distintas experiencias nacionales y regionales, que reportan estos estudios, proporcionan una gran riqueza descriptiva y analítica. No obstante, se concluye que es necesario ampliar las interpretaciones sobre la importancia que tiene el género en los cambios de la cultura política.

\section{PALABRAS CLAVE:}

Acción colectiva | movilizaciones | participación política | mujeres | género

This article presents a review of academic work on the political participation of women in collective action promoted by social organizations, networks, and the movement of women and feminist in Latin America and Colombia, published in the period from 1990 to 2014. This approach gives priority to the analysis of the content of the texts, it focuses on the way as their authors build objects of research, and on the theoretical references and contributions to the knowledge of the relationship gender and collective action. The different national and regional experiences reported by these studies provide a wealth of descriptive and analytical material. However, based on these findings, the article concludes that it is necessary to enlarge the interpretations about the importance that gender changes have in political culture.

\section{KEYWORDS:}

Collective action | mobilizations | political participation | women | gender

Este artigo apresenta um balanço da produção acadêmica sobre a participação política das mulheres em ações coletivas promovidas por organizações sociais, redes e o movimento de mulheres e feministas na América Latina e na Colômbia, publicado no período 19902014. O texto prioriza a análise de conteúdo dos textos, concentra-se em como suas autoras 
constroem os objetos de pesquisa, no referencial teórico e nas contribuições para o conhecimento da relação gênero e ação coletiva. As diferentes experiências nacionais e regionais que relatam esses estudos fornecem uma grande riqueza descritiva e analítica. No entanto, concluiu-se que é necessário ampliar as interpretações sobre a importância do gênero nas mudanças na cultura política.

\section{PALAVRAS-CHAVE:}

Ação coletiva | manifestações | participação política | mulheres | gênero 


\section{Introducción}

Este trabajo parte de una definición de acción colectiva que contempla la existencia de un sujeto social con intencionalidad, que basa su actuación en el cálculo de costos y beneficios, en un proceso de construcción de su identidad política. Aunque en algunas oportunidades los recursos se usan estratégicamente, en otras hay aspectos culturales involucrados en las dinámicas. De ese modo, nuestra revisión tiene en cuenta que la acción colectiva tiene las siguientes características: a) en ella participan tres o más individuos; b) implica un contendor, es decir, que alguien es enfrentado; c) es pública porque vincula a terceros; d) siempre se da por la existencia de un conflicto; y e) generalmente es usada por los subalternos, tal vez porque se constituye en un recurso político valioso que ellos pueden usar contra actores con mayor poder. Las acciones colectivas pueden ser desarrolladas por movimientos sociales, pero también por otros actores, llámense redes de organizaciones o simplemente agrupaciones constituidas por personas que se trazan un objetivo.

La revisión documental sobre la participación política no convencional de las mujeres en América Latina, y especialmente en Colombia, en lo que respecta al activismo de las mujeres por la paz, abarca la producción académica publicada desde 1990 hasta 2014 en libros, artículos y capítulos de libro disponibles en las bibliotecas colombianas y en los portales web de revistas académicas. El análisis de estos trabajos se concentra en captar las particularidades que resaltan estas investigaciones, la forma en que construyen el objeto de investigación, las evidencias empíricas que utilizan, los referentes teóricos que abordan, las estrategias metodológicas que diseñan y el aporte que hacen al conocimiento de la relación género-acción colectiva.

La revisión de la literatura se realizó partiendo de la búsqueda de artículos, capítulos, libros, monografías, trabajos de investigación y tesis de grado, en bibliotecas y centros de documentación de las principales universidades del país. ${ }^{1}$ Se consultaron las bases de datos que contienen publicaciones en Ciencias Sociales ${ }^{2}$ y a partir de los registros hallados se elaboró un listado de los trabajos que incluyeran en su título alguno de los términos: movimientos sociales, acciones colectivas, movilizaciones, protestas y activismo, relacionados con mujeres y feministas en cualquiera de los países de América Latina. Luego, se seleccionaron los que cumplían con el criterio principal del estudio: que hubieran sido realizados después de los noventa, dado que para el caso colombiano es muy importante el período pos-constituyente en el estudio de las formas de movilización de las mujeres. Asimismo, que cumplieran con el criterio de provenir de investigaciones

\footnotetext{
1. Biblioteca Luis Ángel Arango, Universidad Nacional de Colombia, Universidad de Antioquia, Universidad del Valle, Universidad del Cauca, Universidad Javeriana de Bogotá y Universidad de los Andes.

2. Biblioteca digital CLACSO, Academic Search Premier, Fuente Académica, EBSCO, Redalyc y JSTOR.
} 
académicas. Con estos materiales se realizó una exhaustiva lectura que permitiera dar cuenta de los principales enfoques teóricos que utilizan sus autores, de la metodología empleada y del modo en que sus resultados aportan al conocimiento de las acciones colectivas emprendidas y/o pensadas por las mujeres. Para facilitar el procesamiento de esta información se utilizó una rejilla básica de análisis de contenido. Así, el estado de la cuestión que presentamos en este artículo, sirve a los propósitos de la investigación en la que se desarrolló y constituye la base de un estudio más amplio sobre las acciones colectivas de las mujeres y el modo en que el género determina las particularidades de la acción colectiva y el cambio social.

Para el caso colombiano, se identificó la producción académica liderada por profesoras-investigadoras de las diferentes universidades del país, tanto públicas como privadas, de Bogotá, Medellín, Cali y Bucaramanga. En esta literatura se destacan los estudios de sociólogas, historiadoras, antropólogas y politólogas. En esta búsqueda preliminar se identificó que si bien existe una variedad de trabajos y líneas de investigación sobre género y conflicto armado; movimientos de mujeres y feminista; mujeres y actividad política, las acciones colectivas femeninas han sido una preocupación de las académicas y no de los académicos. Una constatación que tendrá que ser analizada con más detalle en otra investigación.

Por ejemplo, Mauricio Archila (2003), uno de los principales investigadores de la protesta en Colombia, plantea que las mujeres son un nuevo actor social en escena, pero sólo dedica algunas páginas de uno de sus libros al activismo femenino. Otros estudiosos de la movilización social en el país resaltan la importancia de las acciones de las mujeres y las analizan como uno de los actores políticos que más se moviliza a finales del siglo XX y principios del XXI (García-Duran, 2006). Sin embargo, en sus estudios no incluyen un análisis de los distintos repertorios que estas utilizan ni las diferencias en sus motivaciones o en los marcos interpretativos. Estos enfoques demuestran que también en Colombia las investigaciones siguen incluyendo a las mujeres como un agregado en las movilizaciones obreras, indígenas, afro descendientes, campesinas, de las movilizaciones por la paz y por los derechos humanos, entre otras, sin analizar los aportes que ellas introducen en estas luchas. Por esto es importante indagar cómo el género influencia determinados comportamientos políticos y contribuye a la configuración una identidad política.

El artículo inicia con la revisión de estudios realizados por académicas de Argentina, Brasil, Colombia, Perú, España, Inglaterra y Alemania, publicados en América Latina; se aclara que esta búsqueda no es exhaustiva y por ello no se referencian todos los materiales que han sido publicados. El ejercicio se concentra en la literatura que trata sobre resistencias, protestas y acciones colectivas impulsadas por mujeres a favor de los derechos políticos, sociales y culturales, y sobre la participación en el movimiento de 
mujeres y feminista. Esta consulta trata de captar las particularidades de este activismo $y$, de algún modo, comparar el caso colombiano con otros de la región.

Después de esta revisión, se muestran los principales aportes de las investigadoras colombianas al conocimiento del activismo que desarrollan las mujeres en el país, los principales problemas que abordan, la calidad de los hallazgos que ofrecen, así como la profundidad de sus análisis, entre otras interpretaciones sobre la organización necesaria para la movilización y los intereses que las motivan. Luego, el artículo se concentra en un tipo específico de movilización en Colombia: las acciones en contra de la guerra o por la paz, la verdad, la justicia y la reparación de las víctimas del conflicto armado. Por último, se aportan algunas reflexiones que suscitó el balance de estudios previos y se establecen algunas líneas para cubrir campos de investigación todavía inexplorados.

\section{Los estudios sobre protestas, movilizaciones y acción colectiva en América Latina}

En los estudios sobre la participación política no convencional de las mujeres en América Latina resalta el carácter interdisciplinario de la literatura publicada. Desde distintas vertientes se ha producido un conocimiento propio sobre los contextos políticos en que se desarrolla la movilización de mujeres. Uno de los aportes más importantes de esta producción y reflexión feminista a los estudios de género es la intersección de otras categorías sociales como la clase, la etnia, la religión y la participación en grupos que defienden los derechos humanos. Estos aspectos suman variables para enriquecer el análisis sobre los cambios que experimentan las mujeres conforme avanza la modernidad. Se genera así un nuevo campo de estudios que produce una amplia bibliografía, y desde una renovación conceptual produce nuevos enfoques para analizar esta diversidad de nuevos actores. No obstante, vale la pena resaltar que en muchos estudios no es clara la diferencia entre movimiento feminista ${ }^{3} \mathrm{y}$ movimiento de mujeres.

En el período que nos interesa (1990-2014) uno de los primeros estudios que se publicó sobre este asunto es el compendio publicado por la colombiana Magdalena León (1994), sobre la participación política de las mujeres en la región: Mujeres y participación política: avances y desafíos en América Latina. En esta obra se presentan los artículos de Lola Luna, Norma Villareal, Virginia Vargas, Nancy Saporta, Jane Jaquette, Marta Lamas, Gioconda Espina, Amy Conger, Sonia Álvarez, Teresa Valdés y María del Carmen Feijoo. Estos análisis académicos sobre el surgimiento del movimiento de mujeres en la región coinciden en que este creció y se desarrolló en países sometidos a regímenes 
autoritarios o con alteraciones internas del orden público, lo que significa que sus organizaciones han actuado decisivamente en la recuperación democrática. Su incursión en la política convencional se hizo para cuestionar los vicios de formalidad y exclusión de los regímenes políticos, hacia finales de los setenta y principios de los ochenta. En estos estudios se explica que el movimiento de mujeres significa un proceso de recalificación de la democracia que no se detiene en el ejercicio pleno de la ciudadanía, sino que está presente en las prácticas de la vida cotidiana (León, 1994).

Para León (1994) este movimiento era menos visible en los años setenta debido a su anclaje en lo privado, pero hacia los años noventa convoca a más mujeres que comparten múltiples iniciativas y formas inéditas de organización. De los sesenta a los noventa se consolidaron en la región fuertes redes en torno a la salud integral y reproductiva, el trabajo doméstico, los DD.HH., la etnicidad, el derecho al aborto y contra la violencia sexual y de género. Por ello, afirma que es pertinente aceptar que el movimiento social de mujeres significa una redefinición del poder político y la forma de entender la política, más que una búsqueda del poder o de la representación en la política formal, es decir, en los partidos políticos, los sindicatos, los gremios y las instituciones políticas, aunque tampoco sea ajena a estos ámbitos. Su acuciosa participación en la vida comunitaria, en la protesta social, en actividades cívicas y hasta en los grupos armados demuestra que el movimiento de mujeres se nutre de formas alternativas de participación política, muchas de ellas de carácter subversivo.

Pero si bien este movimiento integra de manera creativa las solicitudes del género a las reivindicaciones más amplias de la sociedad latinoamericana, ese diálogo no está exento de confrontaciones. Virginia Vargas (1994) se refiere a esa posición contradictoria en la que se desarrolla; aunque insiste en sus aciertos y desaciertos, sus avances e intolerancias, también destaca el dinamismo del proceso. Sonia Álvarez (1994) subraya que la transición de pequeños grupos a un movimiento de amplia heterogeneidad social tiene que afrontar diferencias políticas e ideológicas, que casi siempre se solucionan o reformulan.

Estas autoras reconocen que no se ha logrado la unidad en el movimiento. No obstante, entre la pluralidad de matices y tendencias que allí confluyen parece haber acuerdo sobre el sentido político de sus luchas. Lola Luna (1994), por ejemplo, considera que parte de sus acciones son de naturaleza política, porque tienen que ver con los procesos de cambio que afectan las relaciones de poder entre el Estado y la sociedad. Un proceso que es posible, entre otros factores, por la superación de las dictaduras, la crisis del modelo de desarrollo, el auge del feminismo en América Latina y la atención de las desigualdades de género por parte de los organismos internacionales. Una coyuntura apropiada para la consolidación de su identidad como sujeto político construida a partir de su participación en la lucha por la democracia en organizaciones feministas o de política tradicional. 
A partir de los artículos de esta compilación se concluye que los feminismos crecen en países con regímenes autoritarios o con alteraciones de orden público, pero que en su acción van reconceptualizando la democracia, intentando una nueva cultura política, tal como lo hacen otros sectores subordinados. Así, la participación de las mujeres en el movimiento latinoamericano ha buscado más bien una redefinición del poder político desde la política no formal, con formas alternativas y contenidos que politizan la esfera privada y que se movilizan mediante múltiples formas que reivindican sus propias prácticas como movimiento.

Tres años después de la aparición de este trabajo se publicó el libro Women and Social Movements in Latin America. Power from Below, de Lynn Stephen (1997). Un estudio comparativo que analiza seis casos de activismo de base de mujeres en México, El Salvador, Brasil y Chile. Su autora relata las ideas, experiencias y percepciones de las mujeres que participan en la acción colectiva y explica las condiciones estructurales y los discursos ideológicos que marcan el contexto en el que las mujeres actúan e interpretan sus experiencias. El libro incluye entrevistas a activistas, historias de las organizaciones y los movimientos, y una discusión teórica sobre el género, la identidad colectiva, la antropología feminista y los métodos.

En la primera década del siglo XXI se publica el libro de la chilena Teresa Valdés (200o) De lo social a lo político: la acción de las mujeres latinoamericanas, que describe un proceso de larga duración. Presenta la participación de las mujeres desde las luchas por la independencia hasta sus acciones en los ochenta y los noventa. Este trabajo proporciona una visión global del movimiento de mujeres, pero no ahonda en los hechos históricos que actuaron de contexto ni tampoco en las especificidades de cada país. Sin embargo, muestra a las mujeres participando en conflictos armados anti-imperialistas, en la oposición a las dictaduras militares y en las protestas por la defensa de los derechos humanos. Remarca los cambios en los objetivos del movimiento de mujeres de los años recientes, que parecen concentrarse en la lucha contra la violencia doméstica, en pro de los derechos reproductivos, en la búsqueda del desarrollo con equidad, la participación en las esferas del poder y el cambio cultural.

En 2001, Sonia Álvarez publicó el capítulo "Los feminismos latinoamericanos se globalizan: tendencias de los noventa y retos para el nuevo milenio", en el libro Cultura política y política cultural. Allí señala que la transnacionalización del movimiento feminista ha sido posible gracias a la "ongeización", es decir, al vasto entramado de ONG que componen su organización. La financiación que procedía de las ONG del Norte contribuyó a la coordinación de asuntos a nivel regional y en algunos casos planetario. Aunque está de acuerdo en que la "agenda de género es una agenda sin fronteras", considera que en la situación actual el feminismo está perdiendo capacidad transformadora, puesto que las organizaciones de mujeres en ese momento se conformaban con la institucionalización de los asuntos, sin cambios en la cultura. 
La socióloga peruana Virginia Vargas (2002), desde una "lectura político personal", escribió el artículo "Los feminismos latinoamericanos en su tránsito al nuevo milenio". Distingue tres vertientes del movimiento: la feminista, la de mujeres urbano-populares y la de mujeres adscritas a los espacios más formales y tradicionales de participación política. Estas vertientes se multiplicarán y desarrollarán sus propios objetivos, dinámicas de interrelación y confrontación en los noventa. El texto es un ensayo de interpretación en el que se evalúa la acción del movimiento feminista en las últimas décadas del siglo $\mathrm{XX}$. Para ella, el movimiento ha mantenido una perspectiva subversiva; el proceso de globalización ha permitido entrar en nuevos terrenos de disputa que han nutrido las dinámicas locales; los asuntos de género y de las mujeres se han institucionalizado, muchas mujeres se han vinculado al Estado y todo ello ha implicado ganancias en la capacidad de propuesta, en la profesionalización de sus líderes y en cierto nivel de influencia en las instituciones políticas, pero esto no ha permitido que éstos asuntos se posicionen en los espacios "macro". De paso, en este tránsito se ha perdido la capacidad de movilización, la creatividad e incluso la innovación de otros tiempos. En parte, esto ha acontecido por el retraimiento de la movilización política del feminismo militante, que se ha desplazado a las ONG y a las universidades.

En 2003, se tradujo el trabajo de la socióloga inglesa Máxime Molineux (2003), Movimientos de mujeres en América Latina. Estudio teórico comparado. Para ella, gran parte de los trabajos sobre el movimiento de mujeres ha sido descriptivo y solo hasta hace poco las corrientes teóricas examinan los factores históricos, sociales e institucionales que condicionan el surgimiento de su activismo y las formas genéricas y específicas de identidad colectiva; categorizan y diferencian los tipos de movimientos; analizan la relación entre estos movimientos y la democracia y explican la articulación y transnacionalización de sus agendas y estrategias. Su análisis se concentra en la interacción entre género y política, desde el enfoque del desarrollo y la sociología política. Las cuestiones teóricas desde las que parte tienen un carácter comparativo, que describe cómo los procesos específicos de un país determinado son en gran medida procesos internacionales.

Ese mismo año, la historiadora española Lola Luna (2003), radicada por esos años en Colombia, publicó Los movimientos de mujeres en América Latina y la renovación de la historia. El libro compendia varias ponencias de su autoría y gira en torno a dos premisas: que las luchas de las mujeres están excluidas de los intereses de la historia política y que este tema forma parte de la historiografía de las mujeres. En el tercer capítulo, la autora introduce la discusión sobre la forma en que deben ser analizados los movimientos de mujeres y defiende la idea de interpretarlos como representaciones políticas de la inclusión y de la exclusión. Por último, la autora indaga cómo un movimiento populista articula el discurso de la modernidad sobre las mujeres y construye o reconstruye la identificación mujer/madre. Los líderes reproducen y refuerzan el maternalismo que 
la sociedad asocia con las mujeres y que en muchos casos ellas representan sin mayor cuestionamiento.

Como conclusión, la autora plantea que haría falta una historia del Estado en América Latina que contemple la relación de las mujeres con la política, haciendo visibles las relaciones de género que están presentes en la organización social y política, un asunto que ella introduce al proporcionar una descripción preliminar de las relaciones de género en la construcción del Estado en América Latina y el significado político de las luchas de las mujeres. Luna (2003) resalta que existe un proceso en la región que ha permitido la ampliación de los márgenes de participación que combinan la esfera pública y la privada. Quizás la contribución más importante de este estudio es la construcción de la categoría maternalismo, que destaca la condición de madres de militantes y activistas en las acciones colectivas femeninas. Los casos más destacados son las madres de los desaparecidos por las dictaduras y otras violencias, las organizaciones por la supervivencia y el movimiento feminista que reivindica la maternidad como una práctica feminista. Es decir, toda una gama de mujeres plurales y diversas que incorporan a su acción variadas prácticas discursivas, relacionadas con su condición de madres.

Varios de los estudios sobre las mujeres de América Latina y el Caribe reflejan que ellas han impulsado sus derechos civiles, políticos y sexuales a partir del progresivo reconocimiento e institucionalización de la perspectiva de género, que se inicia con la primera Conferencia de Naciones Unidas para las mujeres en 1975. En estas investigaciones sigue presente la controversia sobre el carácter femenino o feminista de las luchas, si las organizaciones o movimientos que las promueven son de mujeres o feministas y si estos grupos deberían ser autónomos o afiliarse a los partidos políticos. Ante esta situación, Cañizares (2004) plantea que la academia debe concentrarse más en las acciones que en los grupos que las originan, porque está comprobado que ambos defienden tanto las necesidades prácticas, como los intereses estratégicos, dependiendo de las oportunidades políticas que se presenten para las mujeres. ${ }^{4}$ En esa misma línea Murga (2006) plantea que el movimiento de mujeres latinoamericano responde a una ola masiva de movilizaciones de nuevos actores sociales que, en los años setenta y ochenta, entran en la escena política con demandas de reconocimiento de sus identidades y que desde los noventa se agrupan en novedosas formas de organización para reclamar intereses no sólo políticos sino culturales.

De estos cambios en la forma que las mujeres presentan sus demandas se da cuenta en varios de los artículos de la colección De lo privado a lo público: 30 años de lucha

4. La expresión necesidades prácticas e intereses estratégicos fue introducida por Maxime Molineux (1987) para referirse a las diferencias entre las reivindicaciones que hacen las mujeres y las feministas. Para esta autora, las primeras se refieren a los aspectos que facilitarían cumplir con los roles asignados; mientras que los segundos están ligados a los propósitos emanados del esfuerzo consciente de transformación de género. Para ella, no se puede tomar como un hecho la unidad y cohesión de las mujeres sobre asuntos de género: "Esa unidad nunca está dada, siempre hay que construirla”. 
ciudadana de las mujeres en América Latina, editada por Lebón y Maier (2006). Varias autoras reflexionan sobre el significado de la democracia social y política del feminismo para las madres de desaparecidos, mujeres urbano-populares, guerrilleras, sindicalistas, indígenas, lesbianas y afroamericanas. Lo que permite comprender los desafíos y oportunidades que representa el siglo XXI para sus luchas en esta región. También es importante su descripción sobre la historia de los movimientos nacionales y regionales y la materialización de sus logros en los diseños institucionales de políticas públicas.

Para Maier (2006) esta creciente participación de las mujeres en espacios políticos ha creado nuevas representaciones de lo femenino en el imaginario colectivo. Ella afirma que paulatinamente las feministas históricas descubrieron a través de su práctica colectiva de reflexión que lo personal y lo privado se fusionaban. Destaca que en este proceso fueron muy importantes los grupos de autoconciencia para reflexionar sobre la condición de género. En ellos, mediante una metodología de búsqueda colectiva de interlocución y rendición de cuentas entre pares, compartieron sus experiencias personales y gradualmente desnudaron la esencia política del ejercicio (íntimo, privado y público). A través de esta práctica se destejieron las creencias y cuestionaron los dispositivos socioculturales que subordinan a las mujeres.

Otro libro importante es el de la peruana Virginia Vargas (2008): Feminismos en América Latina. Recopila varios artículos de su autoría y presenta un balance del aporte del movimiento a la política y la democracia. Critica las trampas de la representación de las mujeres en el gobierno y plantea los puntos centrales de la agenda feminista, pensando en el rescate de la utopía. ${ }^{5} \mathrm{Al}$ mismo tiempo, desarrolla un análisis sobre la tensión entre “institucionalizadas" y "autónomas" en los feminismos latinoamericanos y se refiere a la construcción del internacionalismo feminista y la participación política de las mujeres, los marcos y las interrelaciones entre lo local, nacional, regional y global.

También incorporamos el libro de la alemana Barbara Potthast (2010): Madres obreras, amantes, que se refiere al protagonismo femenino en la historia de América Latina y que se detiene en los casos de Cuba, Nicaragua, Bolivia y México, para resaltar la participación de las mujeres en las guerrillas de estos países, así como algunas lideresas de gran reconocimiento en lo que la autora denomina las "revoluciones" mexicana, cubana, peronista. También dedica un capítulo especial al "nuevo movimiento femenino" que estaría compuesto por las madres y las mujeres que se han opuesto a las dictaduras militares en el Cono Sur, así como a su participación en el proceso de redemocratización.

\footnotetext{
5. Al respecto, Maier (2006) plantea que en esta etapa inicial "el feminismo se caracteriza por su radicalidad, frescura, transgresión y subversión centrando su atención en los campos de la sexualidad, la reproducción, la violencia de género, la equidad social y económica y -subyacente a todo- la relación entre lo privado y lo público. En este sentido el feminismo situó los derechos de las mujeres, -como señala Jelin (1997)- no únicamente en el terreno de las relaciones de género sino también en la reconsideración de qué es público y qué es privado, lo que implicó una profunda revisión del paradigma civilizatorio" (Maier, 2006: 37).
} 
Por último, otro texto de corte más antropológico es el de Francesca Gargallo (2012): Feminismos desde Abya Yala. Ideas y proposiciones de las mujeres de 607 pueblos de nuestra América: cuestiona al feminismo occidental con preguntas y malestares de las indígenas sobre la forma en que este las ha concebido. Su autora intenta "una reflexión sin conclusiones" acerca de los puntos en común de las luchas indígenas y feministas.

En la revisión de estos y otros estudios ${ }^{6}$ llama la atención el desconocimiento de las luchas de las colombianas, mientras se exaltan los casos de Cuba y Nicaragua por su participación en las guerrillas; la oposición de las mujeres del Cono Sur a las dictaduras que padecieron hasta los ochenta, que involucra acciones de defensa de los DD.HH. y en contra de la crisis económica. Para el caso de Brasil se exploran los cambios en las relaciones feminismo, sociedad civil y Estado. Se destaca la movilización de peruanas, mexicanas y caribeñas en redes internacionales por la garantía de los derechos sexuales y reproductivos y la participación de las indígenas en las revueltas étnicas por los recursos naturales en Ecuador y Bolivia. Una de las principales conclusiones es que el movimiento de mujeres constituye un mecanismo de lucha por la ampliación de la ciudadanía y el reconocimiento y legitimación de la presencia social. En términos de Melucci (1994), el objetivo de sus movilizaciones no es únicamente la lucha por la igualdad de derechos, sino el derecho a ser diferente.

Esto no quiere decir, que el movimiento de mujeres en Colombia sea débil o que los análisis sobre las luchas de las mujeres por los DD.HH., la oposición a la guerra, la movilización por los derechos sexuales, la ampliación de la participación política o el reconocimiento de sexualidades diferenciadas sean insuficientes; más bien demuestra que la producción colombiana tiene escasa visibilidad en el ámbito académico latinoamericano. A pesar de la especificidad de su producción, otras académicas latinoamericanas y los estudiosos de la acción colectiva no suelen referenciar la literatura que se publica en Colombia y escasamente nuestro país es tomado en cuenta para establecer comparaciones con otros de la región. Una hipótesis en este sentido es que la producción del conocimiento es un campo dominado y a pesar de la visibilidad que adquiere una autora como Magdalena León, la producción colombiana sigue siendo periférica, comparada con otras autoras aquí reseñadas.

\section{Los estudios sobre protestas, movilizaciones y acciones colectivas de mujeres en Colombia}

En la búsqueda relacionada con el activismo de las colombianas en el movimiento de mujeres y feminista, su historia, su particularidad, los intereses por los cuales se han movi- 
lizado, al igual que los contextos políticos que han permitido o impedido su participación, las referencias son más amplias que la muestra que aquí reseñamos. Empezamos este recuento en orden cronológico, del mismo modo que lo hicimos con el ejercicio anterior.

El libro de Lola Luna y Norma Villarreal (1993) Historia, género y política: movimientos de mujeres y participación política en Colombia, 1930-1991, reconstruye las luchas del feminismo colombiano desde la vinculación de las mujeres a la Universidad hasta la promulgación de la Constitución política de Colombia en 1991. El estudio muestra la existencia de cinco períodos, que van desde la constitución de las mujeres como sujetos y la caída en la trampa de la ideología maternalista, hasta el cambio de las reglas del juego en las relaciones entre los sexos y la incursión en las redes feministas internacionales.

Dos años después, la socióloga Norma Villareal (1995) publicó el artículo "Mujeres y espacios políticos" en la compilación Las mujeres en la historia de Colombia. En él describe cómo se estructuran las organizaciones de mujeres para incidir políticamente en la propuesta de reforma constitucional que las reconociera como ciudadanas. En este mismo volumen, Olga Sánchez (1995) da cuenta del movimiento social de mujeres que surge en los años setenta, con grupos feministas de diversas tendencias que ponen en la agenda pública el tratamiento de la sexualidad. De acuerdo con la autora, para 1976 se fortalecieron los grupos de Cali, Medellín y Bogotá que trabajaban en las calles, generando debates en algunos círculos sociales. En los años ochenta llegó el I Encuentro Feminista Latinoamericano y del Caribe realizado en Bogotá, que impulsó la movilización y trabajo político de la región. Surge en el país la necesidad de especificar y concretar formulaciones teóricas y estratégicas del feminismo y se empieza a gestar la Red Nacional de Mujeres, la Red Colombiana por los Derechos Sexuales y Reproductivos y la Red de Mujeres del Suroccidente del país, entre otras.

A propósito del surgimiento de estas redes y de la amplia participación de los grupos de mujeres en iniciativas del movimiento de mujeres frente a la agenda política nacional, Martha Londoño (2002), hace un recuento de las principales apuestas para el período 1988-1995. La investigadora destaca las grandes movilizaciones en contra de la violencia, la participación en el proceso de la Asamblea Nacional Constituyente, el trabajo organizativo preparatorio de la IV Conferencia Mundial de las Mujeres de Naciones Unidas en Beijing. Este capítulo se concentra en las acciones de los grupos de Cali y realiza un buen ejercicio de análisis del contexto nacional en el que participan estas activistas. También dedica algunas páginas a mostrar las dificultades que enfrenta el movimiento social en su interlocución con el Estado y lo que ella denomina los logros en la política pública.

En el estudio de tipo documental realizado por Herrera et al. (2005) sobre La construcción de cultura politica en Colombia: proyectos hegemónicos y resistencias culturales, los autores indagan por la particularidad de lo político frente a las identidades de género, analizando la relación entre educación y cultura política. Encuentran que los trabajos coinciden en señalar que la condición política social y económica de la mujer colombiana 
ha estado marcada por factores vinculados a su condición sexual y, que a pesar del reconocimiento jurídico, las leyes mantienen las diferencias discriminatorias. Más que una nueva teoría del Estado y de las relaciones de género, proponen entender determinados contextos institucionales y organizacionales en los que participan las mujeres con el objeto de identificar qué facilita o limita la representación de sus intereses.

En el artículo de María Emma Wills y Diana Gómez (2006) “Los movimientos sociales de mujeres (1970-2005). Innovaciones, estancamientos y nuevas apuestas", se ofrece un breve recuento de la peculiaridad de las acciones colectivas de las mujeres, de la relación entre organizaciones femeninas y movimientos sociales y de las trayectorias desde la esfera social a la política. Las autoras establecen tres períodos de reivindicaciones: 19781988, cuando impugnan las construcciones culturales de lo femenino y lo masculino, se vinculan con la izquierda y se fracturan por la doble militancia. Entre 1988-1998 se recibe el respaldo de las agendas globales para influir en el Estado; y entre 1998-2005 se produce una amplia movilización en contra de la guerra y por la paz, que evidencia la fragilidad para enfrentar las arremetidas de los actores armados, los partidos y el Gobierno para capturar sus agendas.

Después de este escrito, la politóloga María Emma Wills (2007) publicó su tesis doctoral bajo el título Inclusión sin representación. La irrupción política de las mujeres en Colombia 1970-200o. En este libro reconstruye las iniciativas colectivas de mujeres y feministas en la política y en la academia. Muestra el proceso de inclusión en estos espacios públicos y concluye que las mujeres han logrado niveles moderados de inclusión y un bajo grado de representación. La línea de análisis se concentra en la participación formal y por ello describe con más detalle los logros políticos y electorales, que los logros culturales del movimiento de mujeres, aunque se refiera a ciertas formas en que estos dos tipos de participación se retroalimentan.

Al final de la década, la socióloga Doris Lamus publicó varios documentos sobre el movimiento de mujeres en Colombia. Los más conocidos son Lamus (2009): La trasgresión de la cultura patriarcal: movilización feminista en Colombia (1975-1995) y Lamus (2010): De la subversión a la inclusión: Movimientos de mujeres de la segunda ola en Colombia, 1975-2005. En el primero analiza la movilización feminista en el país durante el proceso pre y pos-constituyente, que parece marcar grandes transformaciones en el feminismo colombiano, porque aumentan las demandas por inclusión a través de la política institucional. En el último argumenta que el campo discursivo, definido a partir de las prácticas de mujeres/feministas de la segunda ola, se institucionaliza, construye y reconstruye en interacción con el discurso de Naciones Unidas, las agencias de cooperación y algunas políticas estatales. Este trabajo contribuye al estudio de las luchas de las mujeres al dar cuenta de las interacciones entre lo nacional y lo global, aunque privilegia el proceso regional en Santander y la Costa Caribe. También incorpora en el análisis la teoría de la acción colectiva y de los movimientos sociales desde un enfoque constructivista, tenien- 
do en cuenta que el movimiento como actor político se constituye con un componente discursivo que orienta la acción.

Lamus realiza este análisis desde la integración teórica de tres enfoques: las oportunidades políticas, las estructuras de movilización y los procesos sociales de interpretación de la realidad y asignación de significados, fundamentándose en autores como Alain Touraine y Alberto Melucci. La particularidad de este trabajo radica en que es un análisis desde la militancia de una feminista académica, que confiere un lugar privilegiado a los testimonios de las protagonistas y a los documentos elaborados por las organizaciones sociales.

El último libro que incluimos en este balance es de Norma Villarreal y Lola Luna (2011): Movimientos de mujeres y participación política, Colombia del Siglo XX al XXI, que actualiza el publicado por estas autoras en 1993. En la nueva versión se incluye el análisis del movimiento de mujeres y feminista desde dos enfoques: la incidencia y la resistencia. Es decir, las acciones orientadas a la institucionalización de las agendas (políticas públicas, programas de superación de la desigualdad, etc.) y aquellas dirigidas a denunciar la violencia que produce el conflicto armado y poner en cuestión las acciones bélicas de los actores armados, tanto insurgentes como legales. También señalan que las demandas por servicios psicológicos y jurídicos que hacen las víctimas de la violencia en Colombia estarían acercando a las mujeres populares con las de sectores medios, profesionales, de ONG y académicas. Además, destacan que en estos tiempos se han logrado consolidar múltiples alianzas entre las mujeres y "otras minorías", como jóvenes, afrodescendientes e indígenas, que han contribuido a mejorar la incidencia en el Estado. No obstante, esta dispersión de intereses parece estar retrasando las actuaciones de las mujeres para obtener logros más democráticos y libertarios, tales como los que se propuso el feminismo en otros tiempos.

\section{Los estudios sobre el activismo por la paz y en contra de la violencia en Colombia}

Como plantean algunas de las autoras mencionadas, desde los años noventa la agenda política del movimiento de mujeres en Colombia incluye demandas para la resolución del conflicto armado, la construcción de paz y la atención de las víctimas. Es en este aspecto que las organizaciones de mujeres y organismos internacionales concentran las denuncias y el protagonismo del movimiento. Son múltiples las publicaciones de este orden, en páginas web y blogs de las organizaciones de mujeres, que han recibido apoyo tanto de agencias internacionales como de ONG globales, para dar cuenta de las iniciativas de paz y, sobre todo, para denunciar la violación de derechos humanos y de violencia contra la mujer en medio del conflicto. No obstante, como criterio para la elaboración 
de este estado de la cuestión, a continuación relacionamos la producción bibliográfica académica, tal como se hizo en los anteriores acápites, por lo tanto, sugerimos a los interesados en este asunto someter los materiales que producen las organizaciones al tratamiento de fuentes documentales. ${ }^{7}$

El activismo de las mujeres a favor de la paz y en contra de la violencia que ejercen los actores armados contra la población civil se ha convertido en un campo de interés entre estudiantes de pregrado, maestría y doctorado de diferentes disciplinas de las ciencias sociales en el país y el exterior. Abundan las monografías, los trabajos de investigación de maestría y tesis doctorales que se interesan por los casos de mujeres indígenas, campesinas, afrodescendientes, entre otras víctimas, que deciden integrarse a las grandes redes de mujeres para denunciar los atropellos contra sus comunidades en el marco del conflicto armado, o de aquellas que exigen el intercambio humanitario de secuestrados por guerrilleros presos, la solución negociada del conflicto armado, o la participación de las mujeres en las mesas de negociación con las AUC, las FARC o el ELN, entre otras reivindicaciones. No obstante, dado el interés de esta investigación por la literatura publicada, tampoco se tienen en cuenta estos materiales. A continuación mostramos un balance de libros y capítulos publicados en revistas.

En 1997, Norma Villarreal publicó "Mujeres y madres en la ruta por la paz". Un artículo que se interesa por las movilizaciones en favor de la paz que captaron la atención de los medios de comunicación durante la crítica situación del país a mediados de los noventa: las protestas de las madres por la prestación del servicio militar de los bachilleres en los frentes de guerra, las movilizaciones de las madres de los soldados secuestrados por la guerrilla en Las Delicias, reclamando la liberación de sus hijos, y la marcha a Mutatá para buscar una solución negociada al conflicto armado. La autora sitúa estas tres movilizaciones en continuidad con la tradición de las mujeres en la lucha por la paz, que se remonta a su participación en la Marcha del Silencio en contra de la violencia partidista, convocada por Jorge Eliécer Gaitán en 1940. Villarreal rescata la influencia del movimiento feminista en la crítica a la guerra y a los actores armados y en la búsqueda de alternativas pacíficas. En su comentario a esta ponencia, publicada

7. Algunos textos se han publicado con auspicio institucional, como Pilar Arango (1995) Mujer y conflicto armado. Elementos para la discusión. Secretaría de Mujer y Género de Bogotá. Se destaca el esfuerzo de las organizaciones y sus activistas por publicar sus reflexiones en revistas y documentos propios, cuatro ejemplos son ilustrativos de este estilo: Rocío Pineda (1997). Mujeres entre la guerra y la paz: Lisístratas colombianas a las puertas de un nuevo milenio, Conferencia presentada en el V Congreso Nacional de la Unión de Ciudadanas de Colombia, Cali; Mujeres Pazíficas (2002): Que nuestro cuerpo no sea escenario de guerra, Santiago de Cali; OFP (2004):Documento propositivo en construcción. Memorias I. Movimiento social de mujeres en contra de la guerra, Barrancabermeja, Organización Femenina Popular; Sánchez, Olga A. (2006). Nuevas formas de resistencia civil de lo privado a lo público: movilizaciones de la Ruta pacifica 1996-2003. Bogotá: Ruta Pacífica de la Mujeres. Asimismo, los Organismos Internacionales también publican los resultados de las consultorías que promueven en el país. Por ejemplo, ACNUR (2001) Las Comunidades de Paz: una reflexión sobre la equidad de género; Prieto, Patricia y Tamayo, Luz M. (2004). Las mujeres colombianas en busca de la paz: una aproximación a sus iniciativas y propuestas implementando la resolución 1325. Bogotá: Unifem. 
en la misma compilación, Mauricio Archila (1997) apoya y complementa varios de los argumentos de Villarreal, al tiempo que critica su visión homogéneamente pacifista de las mujeres. Pues a su juicio, en el escrito no se establece las diferencias entre las mujeres guerreras, las guerrilleras o las guerreristas. Asimismo, para Archila, la autora reduce la violencia a una expresión del autoritarismo y el dominio patriarcal.

Por la importancia de la negociación que se estaba adelantando entre el Gobierno nacional y las FARC, varias estudiosas reflexionaron en torno a la paz y la creciente incorporación de las mujeres en organizaciones y colectivos de víctimas, de defensa de los derechos humanos y en amplias redes que se manifestaban a favor de una solución negociada del conflicto armado. Sobre este asunto la historiadora Magdala Velásquez (200o) escribió el artículo "Anotaciones para una postura feminista en torno a las mujeres, la guerra y la paz", en la revista Nova \& Vetera. En él proporciona una mirada a la herencia de las luchas por la paz, hace una aproximación al concepto de paz y muestra los vínculos entre el feminismo y el pacifismo; desde una perspectiva feminista se remonta a los orígenes de la guerra; presenta algunas precisiones sobre las características de la guerra postmoderna; denuncia la condición de las mujeres en las guerras contemporáneas; plantea el papel de las Naciones Unidas frente las mujeres y la paz y, finalmente, concluye con un panorama sobre las posibilidades para la paz en Colombia. Empieza por sintetizar los logros más importantes del proceso, sin desconocer las dificultades y carencias del mismo, entre otras, la escasa vinculación de las organizaciones sociales en la negociación entre el Estado y de la insurgencia.

La revista En otras palabras, del Grupo Mujer y Sociedad de la Universidad Nacional y la Corporación Casa de la Mujer (2001), dedicó su edición No. 8 al tema de las mujeres, violencias y resistencias. Dada la particularidad de los trabajos, en este número confluyen las voces de desplazadas, viudas, excombatientes que participan en diversas iniciativas de paz, con los análisis académicos de Donny Meertens, Magdala Velásquez y Florence Thomas, de feministas militantes en organizaciones sociales, como María Eugenia Vásquez, Olga Amparo Sánchez y Angélica Bernal. Todas las contribuciones señalan, desde su original análisis, el compromiso de las mujeres por la paz, a pesar de los obstáculos que impone la lógica de la guerra y de los graves efectos que esta ha tenido sobre las mujeres.

Dos años después, Gloria Tobón y María Martínez (2003) editaron el libro: El tiempo contra las mujeres. Debates feministas para una agenda de paz, que presenta diferentes reflexiones teórico-prácticas de mujeres que se plantean formas para oponerse a la violencia, la discriminación y la exclusión. Algunas desde prácticas y perspectivas personales, otras desde el apoyo colectivo, político y cultural, para buscar alternativas que les permitan construir agendas que incorporen los derechos de las mujeres. Entre los artículos más importantes están: "Afirmar la vida, develar la guerra: las agendas de paz desde las mujeres”, de Gloria Tobón; “Resistencia y devenir mujer” de Marta López; 
"Fragmentos de experiencias de paz de mujeres", de Magdala Velásquez; “Tejiendo vida en contextos de muerte" de María Beatriz Montoya y "Mujeres reconstruyen la vida en medio del conflicto" de Yusmidia Solano. La mayoría de estos trabajos son reflexiones sobre el trabajo de investigación que cada una de las autoras había realizado y que tienen la pretensión de hacer aportes para una agenda del movimiento feminista a la paz.

De ese mismo estilo son los productos de académicas y activistas de DD.HH., como Alejandra Miller (2005) profesora de la Universidad del Cauca y coordinadora de la Ruta Pacífica en ese departamento. En su artículo "Palabras y resistencias de mujeres del Putumayo en el contexto del conflicto armado colombiano", interpreta las historias de vida de mujeres desplazadas del Putumayo y describe la transformación que sufren los roles femeninos en el proceso de adaptación a la condición de desplazadas por la violencia. Resalta la doble victimización que padecen y cómo se acomodan para responder ante los nuevos compromisos que les impone la reconfiguración de la familia en el lugar de recepción. El texto se concentra en las situaciones que comentan las entrevistadas y realiza importantes interpretaciones sobre la naturalización de la violencia de género y la escasa distinción entre los diferentes tipos de violencia, la reflexión de las mujeres acerca de la organización que han logrado con otras víctimas, el reconocimiento obtenido por las instituciones y la solidaridad entre las mujeres como respaldo a sus capacidades y liderazgos diferenciados.

En 2007, la socióloga Norma Villarreal publicó el artículo “Colombia, violencias, conflicto armado y resistencias de género: las apuestas de una cartografía de la esperanza”. Resalta la capacidad de movilización de las mujeres que se organizan a pesar de la intensificación del conflicto armado y de la violencia en sus territorios. Afirma que las mujeres hacen un importante aporte a la construcción de la paz a partir de las acciones colectivas de resistencia no violenta y de oposición a la violencia estructural. Sus acciones inciden en la disminución del impacto del conflicto armado en sus vidas desde un trabajo local. Apelan a estrategias poco convencionales, pero efectivas, para superar la condición de inequidad y pobreza en sus comunidades, que se agrava por la violencia.

En este estudio se analizan 53 iniciativas en Cauca, Chocó y Nariño, se destacan las productivas, las comunitarias, las de promoción y capacitación de liderazgos. La explicación que ofrece sobre la orientación de las acciones recurre a una sugerente definición de indicadores y objetivos que han trazado las mismas mujeres. Por último, el escrito destaca sus aportes a la resistencia pacífica, la protección de la vida, la capacidad de reconocimiento de otros para integrarlos en sus objetivos, las estrategias para enfrentar la pobreza, empoderarse y convertirse en ciudadanas. Así como la comprensión de sus problemas como asuntos públicos y la relevancia de la cultura propia y la valoración de sus particularidades.

En 2007 y 2008 se publicaron tres artículos de María E. Ibarra, el primero llamado "Acciones colectivas de las mujeres en contra de la guerra y por la paz en Colombia" 
(2007a); "Transformaciones y fracturas identitarias de las mujeres en las acciones colectivas por la paz" (2007b); “ "Guerrilleras y activistas por la paz en Colombia: incursión política y rupturas identitarias" (2008).

El primero describe y analiza las principales acciones de dos redes de mujeres en Colombia: La Ruta Pacífica de las Mujeres y la Iniciativa de Mujeres Colombianas por la Paz, IMP. En el análisis se recurre a las categorías de género y acción colectiva para poner en evidencia cómo esta participación política apoya otros procesos emancipatorios que implican interacciones estratégicas. También se explica cómo las acciones femeninas desafían el poder autoritario de los grupos armados y afirman su liderazgo en los procesos de resistencia e interlocución frente a estos y el Estado. El segundo se concentra en mostrar los cambios y las permanencias de la identidad de género de quienes se movilizan. Los hallazgos hechos en el seguimiento a varias trayectorias de vida de las integrantes de organizaciones de víctimas, étnicas y populares, sugieren que sus identidades se modificaron en la militancia política, en parte por la influencia que reciben de líderes feministas y sindicalistas, las cuales trabajan por el empoderamiento femenino. El tercero establece la comparación de estos procesos entre quienes optaron por la militancia en los grupos armados y aquellas que se vincularon a las organizaciones de paz.

En 2009, Ibarra actualiza algunos de los hallazgos encontrados en la investigación anterior y realiza algunas precisiones de contexto para presentar la contribución que hacen las mujeres a la creación de una cultura de paz, desde la reinterpretación de la maternidad biológica y la politización del dolor que produce la violencia. Estos hallazgos fueron publicados en el libro Victimas invisibles, conflicto armado y resistencia civil.

A la anterior producción, se suma el artículo de Patricia Ramírez (2009): "El movimiento de mujeres frente al conflicto armado en Colombia y la construcción de paz 1998-2008". Ella describe las principales movilizaciones en las que han participado la Ruta pacífica de las mujeres y la IMP, recurriendo a la voz de sus protagonistas. También la socióloga Mary Luz Alzate (2010) analiza las interpretaciones y aportes sobre las acciones colectivas frente a la violencia y el conflicto armado en Colombia. Esta autora identifica que el conflicto armado genera un doble efecto sobre el movimiento: dinamiza la movilización y a la vez la desarticula por amenazas y violencia contra los líderes de las organizaciones, en este mismo sentido los actores se identifican como defensoras de Derechos Humanos o con su situación de víctimas. Para la autora, en la primera década del 2000 es cuando figuran las acciones de mujeres como víctimas de la violencia, y se gestan procesos organizativos que alimentan los estudios e investigaciones sobre las mujeres en el país.

En 2011, se publican los resultados de la investigación Acciones colectivas de las mujeres por la verdad, la justicia y la reparación en Colombia, cuyo eje de análisis es la activa movilización que desencadenó el proceso de negociación del gobierno con las Autodefensas Unidas de Colombia entre 2003 y 2009. Entre otras cuestiones, este proceso 
compromete a las organizaciones de mujeres en la discusión sobre las apuestas de la justicia transicional, que serán tenidas en cuenta en la formulación de la Ley de Justicia y Paz de 2005. Una de las conclusiones más importantes de este estudio es que hay una importante influencia feminista en el reclamo por redistribución y reconocimiento para las víctimas del conflicto armado y que estas acciones colectivas empiezan a modificar las reglas institucionales y el modo en que el Estado atiende los derechos humanos de las mujeres (Ibarra, 2011a; 2011b).

Para finalizar esta sección, hacemos referencia a las contribuciones del Grupo de Memoria Histórica (2010 y 2011), al destacar los relatos de las víctimas de la violencia en el país en una serie de libros que describen los roles que desempeñan las mujeres durante los asedios a los que han sido sometidas sus comunidades y el liderazgo que han ganado en la defensa de los derechos de las víctimas en el país. Algunos ejemplos son: La masacre de Bahía Portete. Mujeres Wayuu en la mira y Mujeres que hacen historia. Tierra, cuerpo y política en el Caribe colombiano. No obstante, esta importante literatura producida sobre el tema todavía hay varios aspectos que requieren mayor profundidad analítica para explicar las conexiones entre el feminismo y el pacifismo y la comparación de las acciones colectivas de mujeres y otros grupos que se movilizan por la paz y en contra de la guerra en Colombia.

\section{Reflexiones finales}

El análisis de las fuentes consultadas permite plantear unas primeras reflexiones sobre la forma en que ha sido estudiada la participación de las mujeres en acciones colectivas. A continuación enunciamos los hallazgos y las contribuciones más importantes de estas investigaciones y las vetas que descubren para el análisis de este proceso social.

Tal como se deriva de varios de los estudios reseñados sobre la participación de las mujeres en América Latina, las académicas otorgan gran importancia al contexto político en el que surgen sus movilizaciones, pues este ha propiciado que se formulen exigencias de ampliación de la democracia y la mejora de su representación en las instituciones del Estado. Su atención también se ha centrado en el análisis del cambio en los intereses por los que se movilizan; al pasar de las grandes utopías de transformación de la sociedad patriarcal a incorporar demandas por derechos sexuales y reproductivos, el derecho al aborto, la eliminación de la violencia contra las mujeres, entre otros. Otras estudian el modo en que la incorporación de nuevos asuntos a sus luchas modifican las denuncias que hace el movimiento de mujeres, al tiempo que otras ponen su foco en la ampliación de las formas de protesta y en la estructuración de redes y alianzas que revitalizan el movimiento de mujeres. 
Otro hallazgo importante, que aportan estos estudios, es haber descifrado las particularidades que presenta ese actor político denominado mujeres; captar el cuestionamiento que hace de los dispositivos socioculturales que las subordinan y plasmar en ricas descripciones las formas inéditas de movilización, recurriendo a repertorios añejos, actualizándolos o innovando en otras formas de acción colectiva ligadas a los contextos o aprovechando la estructura de oportunidades políticas. Las autoras destacan la amplia participación política de las mujeres en organizaciones y redes que se unen al movimiento social, y en este la confluencia de una gran heterogeneidad de integrantes, procedentes de distintos sectores y con una vasta gama de características sociológicas y culturales. Algunas de estas autoras afirman que esta amplitud de variables ha impedido la unidad de reivindicaciones, pero al tiempo ha generado una inmensa solidaridad entre las mujeres que lo conforman. Ello habría permitido que las luchas se libren en un escenario más global y menos local, pero también que se debiliten las grandes utopías del movimiento, puesto que ahora lo importante estaría cediendo espacio a lo urgente; dado el apoyo del movimiento a las demandas de sus afiliadas.

De acuerdo con sus reflexiones, esta multiplicidad de activistas y de organizaciones ha permitido la renovación de los repertorios de acción del movimiento en el despliegue de sus acciones colectivas. Una de las experiencias más destacadas en esas modalidades de acción se nutre de su condición de madres, un recurso valioso que es usado estratégicamente tanto en su protección como militantes, como en la movilización de más mujeres que se convencen de la importancia de actuar por sus hijos.

En estos análisis, las autoras valoran el apoyo de la cooperación internacional, que contribuye a capacitar más mujeres y ayuda, de manera notable, a aumentar la conciencia de la subordinación que ellas sufren. No obstante, critican su interferencia en la construcción de la agenda del movimiento. Igualmente, se destaca su censura a la representación que se arrogan algunas de las líderes, que habrían permitido su cooptación y la negociación de las reivindicaciones del movimiento con el Estado. Este diagnóstico propició la revisión académica de lo que estaba aconteciendo en el quehacer del feminismo militante y de las mujeres agrupadas en el movimiento. Para varias de estas estudiosas, la crítica feminista desde las universidades distanció la academia del activismo político y generó un resquebrajamiento de las buenas relaciones que estos dos espacios habían mantenido. Sin embargo, ellas reconocen que esa revisión no sólo mejoró la comprensión de los nuevos retos del movimiento, sino la incorporación de nuevas formas de actuar y de liderar las luchas por la igualdad. De ese modo, las académicas plantean sus posiciones políticas y en sus escritos superan la autocomplacencia con las acciones de los movimientos de mujeres y feminista, de los que varias de ellas son activistas, y desde una perspectiva auto-etnográfica analizan su experiencia, un asunto que cada vez adquiere mayor importancia en las interpretaciones sobre la acción colectiva. 
Buena parte de estas investigaciones reconoce la progresiva institucionalización de la perspectiva de género en la acción de las instancias internacionales, en parte por la labor que ha liderado Naciones Unidas en las diferentes conferencias dedicadas a las mujeres. Así mismo, es notable el valor que le otorgan a esta labor en la materialización de las reivindicaciones del movimiento hacia el diseño institucional de políticas públicas con enfoque diferencial.

En pocas referencias consultadas se recurre a la reinterpretación histórica del activismo en el que se inmiscuyen las mujeres. No obstante, los citados aquí constituyen un valioso aporte de las historiadoras feministas que han incorporado la perspectiva de género en sus análisis y que ahora "descubren" aspectos que fueron "invisibilizados" por los historiadores en sus recuentos y en la narración de los acontecimientos. Asimismo, es relevante la contribución del enfoque postcolonial sobre la participación de las mujeres, puesto que él ha permitido a las autoras incorporar a sus reflexiones la voz y el sentido que los subalternos le otorgan a sus experiencias reivindicativas. Estos tratan de escapar a los enfoques tradicionales, que utilizan marcos teóricos y métodos de investigación rígidos, para sugerir nuevas formas de entender, por ejemplo, la participación de las mujeres indígenas, de las afrodescendientes o de las lesbianas.

De forma particular, en la sección que se refiere a Colombia se destacan hallazgos como los siguientes: las colombianas históricamente han tenido una activa participación en distintas organizaciones sociales. Esa experiencia ha contribuido a estructurar sus reivindicaciones en momentos cruciales de la historia del país. Por ejemplo, su vinculación a las discusiones para redactar la Constitución Política de 1991 u oponerse a una Ley, como la de Justicia y Paz, que se tramitaba en el Congreso de la República como marco para la desmovilización paramilitar en 2005. Del mismo modo, ese ejercicio participativo ha permitido su vinculación a redes, la consolidación de grandes alianzas y su asistencia a eventos internacionales, como las conferencias de Naciones Unidas. Teniendo presente estos hechos, a las académicas les llama la atención el carácter confrontador que tiene el activismo feminista, más que el femenino. Resaltan que el primero evita las transacciones institucionales, recibir favores y aceptar reformas puntuales; mientras que el segundo, el de las mujeres, dedica esfuerzos a la incidencia política en la formulación de políticas, conseguir recursos y aliviar necesidades asociadas a los roles de género femenino. Para el primero, tal como señalan estas autoras, es indispensable mantener la utopía de transformación, lo que requiere fortalecer a sus activistas teóricamente, dotarlas de herramientas que les permitan seguir transgrediendo los estereotipos de género.

Por lo anterior, en sus análisis, varias de estas autoras se detienen en la importancia del discurso que han incorporado las militantes de distintas organizaciones a sus reivindicaciones. Este nuevo discurso se apropia de los términos que usan las agencias y organismos de cooperación internacional. Para estas analistas, esto comporta graves implicaciones en la estructuración de las demandas del movimiento, porque como lo 
plantean otras autoras latinoamericanas, ello institucionaliza la agenda, la enmarca en la acción del Estado y le hace perder autonomía al feminismo, como práctica.

En la última parte, dedicada a la mirada de las autoras sobre el rol que han asumido las mujeres en el movimiento por la paz en Colombia, adquiere importancia el análisis que proponen de la emergencia de un sujeto que defiende la negociación política del conflicto armado. Se destaca también su foco en la relación mujeres y paz, desde una perspectiva que supera los esencialismos para proponer que la victimización habría dado argumentos tanto a las mujeres de base como a las viejas militantes para oponerse a la militarización, al belicismo y a la guerra como institución patriarcal.

Un asunto que apenas se insinúa en estos estudios es el de las trayectorias de las activistas. Nuestra propia experiencia nos ha demostrado que este es un filón que proporcionaría importantes respuestas al análisis de la acción colectiva. Por tanto, se sugiere a los futuros investigadores el estudio de esos recorridos, de esas historias de vida y de los tipos de liderazgo que han surgido en su lucha por la defensa de los DD.HH., a favor de la paz y en contra de la guerra. Dada la riqueza de experiencias subjetivas que han tenido quienes se movilizan en Colombia, es importante dar cuenta de cómo se están forjando esos liderazgos, y de qué modo esa incursión en la arena política estaría modificando las identidades personales, sociales y culturales de esas mujeres que se convierten en líderes, en el ámbito local, y se vuelven visibles globalmente.

Es recurrente que las autoras presenten el caso colombiano como excepcional, sin demostrar la singularidad de los hechos que aquí acontecen ni de las razones que los motivan. En varios documentos se desconocen los antecedentes de las luchas de las mujeres en otras latitudes y el modo en que estas influencian y fortalecen tanto al movimiento feminista, al movimiento de mujeres y, de manera particular, sus acciones a favor de la paz y por la defensa de los derechos humanos en nuestro país. En este aspecto, se sugiere emprender estudios comparativos que partan en primera instancia de los aportes académicos existentes y recurran a nuevas fuentes de información como la que generan las propias organizaciones en sus páginas web, blogs y enlaces asociados que publicitan sus luchas.

De esta revisión de estudios previos también se deriva que el interés por estudiar la participación política no convencional se ha ido renovando y cada vez se incorporan nuevos elementos que ayudan a comprender las tensiones que impone el género en la participación política y los cambios que ha propiciado en la cultura política. No obstante este hecho y la reconocida trayectoria política de algunas de las autoras citadas, quienes se reconocen como feministas, estos estudios se concentran en la participación femenina y no establecen comparaciones con la participación de los varones. También llama la atención que la mayoría de los casos se tratan de forma exploratoria, aunque existan antecedentes para avanzar en trabajos más descriptivos o incluso explicativos y comparativos. 
La indagación realizada para distintas investigaciones sobre la acción colectiva de mujeres nos permite aseverar que lo anterior ocurre, en parte, porque la participación de las mujeres, en un buen número de estudios, sigue siendo tratada como un agregado. En las movilizaciones afros, indígenas, campesinas, obreras, pacifistas, ecologistas, estudiantiles y de víctimas, entre otras, se reconoce su amplia participación y capacidad de movilización, pero no los cambios que introduce el componente de género en ese activismo. Los estudios sobre acción colectiva en el país escasamente dan cuenta de las diferencias del proceder de los grupos de mujeres en relación con otros actores que se movilizan. Por ello, este balance intenta mostrar que, a pesar de la abundancia de estudios, todavía se requieren más y mejores análisis del aporte de las mujeres a la renovación de las formas de acción colectiva y a los cambios en la cultura política.

\section{Referencias}

ACNUR. (2001). Las Comunidades de Paz: una reflexión sobre la equidad de género. Colombia. Disponible en: http://www.acnur.org/Pdf/o201.pdf?view=1.

ÁLVAREZ, S. (2001). “Los feminismos latinoamericanos 'se globalizan': tendencias de los 90 y retos para el nuevo milenio”. En: Escobar, A. et al. (eds.). Cultura política y política cultural (pp. 345-380). Bogotá: Taurus-ICANH.

. (1994). "La (trans)formación del(los) feminismo(s) y la política de género en la democratización del Brasil”. En: León, M. (ed.) Mujeresy participación política: avancesy desafios en América Latina (pp. 229-290). Bogotá: Universidad de los Andes y Tercer Mundo.

ALZATE, M. (2010). "Interpretaciones y aportes recientes sobre las acciones colectivas frente a la violencia y el conflicto armado en Colombia”. En: Estudios Sociales. 18(36), 35-55.

ARANGO, P. (coord.) (1995) Mujery conflicto armado. Elementospara la discusión. Bogotá: Secretaría de Mujer y Género.

ARCHILA, M. (2003). 25 años de luchas sociales en Colombia 1975-200o. Colombia: Cinep.

. (1997). "Lisístrata, o mujeres colombianas en búsqueda de la paz”. En: Arango, Luz G. (ed.). La crisis sociopolítica colombiana. Un análisis no coyuntural de la coyuntura (pp. 397-405). Bogotá: CES- Universidad Nacional de Colombia.

CAÑIZARES, A. (2004). “Movimientos de mujeres en América Latina”. En: Millán, C. y Estrada, A. ( eds.) Pensar (en) género. Teoría y práctica para nuevas cartografías del cuerpo (pp.156-169). Bogotá: Instituto Pensar - Universidad Javeriana.

FEMENÍAS, M. L. (comp.) (2002). Perfiles del feminismo iberoamericano. Buenos Aires: Catálogos. GARCÍA-DURÁN, M. (2006). Movimiento por la paz en Colombia 1978-2003. Bogotá: CINEP. 
GARGALLO, F. (2012): Feminismos desde Abya Yala. Ideasy proposiciones de las mujeres de 607 pueblos de nuestra América. Bogotá: Ediciones desde abajo. . (2004). Las ideas feministas latinoamericanas. México: Universidad de la Ciudad de México.

. (2002). "El feminismo múltiple: prácticas e ideas feministas en América Latina". En: Femenías, M. (ed.). Perfiles del feminismolatinoamericano (pp.103-130). Buenos Aires: Catálogos. Grupo de Memoria Histórica. (2010) La masacre de Bahía Portete. Mujeres Wayuu en la mira. Bogotá: Taurus.

. Mujeres que hacen historia. Tierra, cuerpo y política en el Caribe colombiano. Bogotá: Aguilar, Altea, Taurus, Alfaguara S.A.

Grupo Mujer y sociedad y la Corporación Casa de la Mujer. (2001). En otras palabras. Mujeres violencias y resistencias. (8).

HERRERA, M. et al. (2005). La construcción de cultura politica en Colombia:proyectos hegemónicos y resistencias culturales. Bogotá: Universidad Pedagógica Nacional.

IBARRA, M. E. (2011a): “Acciones colectivas de mujeres por la verdad, la justicia y la reparación”. En: Reflexión política. 14 (25), 136-150.

. (2011b): "Mujeres, verdad, justicia y reparación en Colombia". En: Univérsitas Humanistica (72), 247-273.

. (2009). "Mujeres y cultura de paz en Colombia”. En: Bondía, D. y Muñoz, M. (eds.). Victimas invisibles, conflicto armado y resistencia civil (pp. 285-314). Barcelona: Huygens.

. (2008). "Guerrilleras y activistas por la paz en Colombia: Incursión política y rupturas identitarias”. En: Pensamiento psicológico (4), pp. 56-76.

. (2007). Trasformaciones identitarias de las mujeres como resultado de su participación política en las guerrillas y en las acciones colectivas por la paz en Colombia. Madrid: Universidad Complutense de Madrid, Tesis doctoral.

. (2007a). "Acciones colectivas de las mujeres en contra de la guerra y por la paz en Colombia". En: Sociedad y Economía (13), 65-85. . (2007b). "Transformaciones y fracturas identitarias de las mujeres en las acciones colectivas por la paz”. En: Revista la Manzana de la Discordia. 2 (4), 73-84.Jelin, E. (1997). “Engendering Human Rights”. En: Dore, E. (ed.). Gender Politics in Latin America (pp. 65-83). New York: Monthly Review Press.

JAQUETTE, J. (1994) (ed.) The Women's Movements in Latin America: Participation and Democracy. Winchester, Mass.: Unwyn Hyman. 
. (1989) (ed.) The Women's Movements in Latin America: Feminism and Transition to Democracy. Boston: Unwyn y Hyman.

LAMUS, D. (2010). De la subversión a la inclusión: Movimientos de mujeres de la segunda ola en Colombia, 1975-2005. Bogotá: ICANH.

. (2009). "La trasgresión de la cultura patriarcal: movilización feminista en Colombia (1975-1995)". En: La Manzana de la discordia. Universidad del Valle. 4 (2).

LEBON, N. Y MAIER, E. (Coord.) (2006). De lo privado a lo público: 30 años de lucha ciudadana de las mujeres en América Latina. México: Siglo XXI.

LEÓN, M. et al. (1993). Espacio y tiempo: las luchas sociales de las mujeres latinoamericanas. Clacso: Buenos Aires.

LEÓN, M. (1994). Mujeres y participación política: avances y desafíos en América Latina. Bogotá: Universidad de los Andes y Tercer Mundo.

LONDOÑO, M. (2002). "Movimiento de mujeres, feminismo y proyecto político en Cali". En: Castellanos, G. y Accorsi, S. (comp.). Género y sexualidad en Colombia y Brasil (pp.127-172). Cali: Manzana de la Discordia editores, CEGMS - Universidad del Valle.

LUNA, L. Y VILLARREAL, N. (1993). Historia, género y política: movimientos de mujeres y participación política en Colombia, 1930-1991. Seminario Interdisciplinar Mujeres y Sociedad. Barcelona: Comisión Interministerial de Ciencia y Tecnología.

LUNA, L. (2003). Los movimientos de mujeres en América Latina y la renovación de la historia. Cali: Universidad del Valle.

. (1994). "Estado y participación política de las mujeres en América latina una relación desigual y una propuesta de análisis histórico”. En: León, M. (ed.). Mujeres y participación política: avances y desafíos en América Latina. (pp. 29-44). Bogotá, Universidad de los Andes y Tercer Mundo.

MAIER, E. (2006). “Acomodando lo privado en lo público” En Lebon, N. y Maier, E. (Coord.) De lo privado a lo público: 30 años de lucha ciudadana de las mujeres en América Latina (pp. 2947). México: Siglo XXI. . (1997). Dilemas de los feminismos latinoamericanos. Tijuana: El Colegio de la Frontera Norte.

MELUCCI, A. (1994). "Asumir un compromiso: identidad y movilización en los movimientos sociales”. En: Zona abierta. (69), 153-180.

MILlER, A. (2005). "Palabras y resistencias de las mujeres del Putumayo en el Contexto del Conflicto Armado Colombiano" En: Convergencia 12 (37), pp.85-114.

MOLINEUX, M. (2003). Movimientos de mujeres en América Latina. Estudio teórico comparado. Madrid: Cátedra. 
(1987). "Movilización sin emancipación? Intereses de la mujer, el Estado y la revolución: El caso de Nicaragua”. En: Núñez, O. et al. (ed.). La transición difícil. La autodeterminación de los pequeños paises periféricos. (pp. 341-360). Managua: Editorial Vanguardia.

MUJERES PAZÍFICAS. (2002). Que nuestro cuerpo no sea escenario de guerra. Santiago de Cali: Mujeres Pazíficas.

MURGA, F. A. (2006). Los movimientos sociales en América Latina (1980-2000): una revisión bibliográfica. En:Polis:Investigacióny Análisis Sociopolítico y Psicosocial.;2(2), 163-196. Disponible en: http://redalyc.uaemex.mx/redalyc/src/inicio/ArtPdfRed.jsp?iCve=7262020. Consultado: 1 de febrero de 2011.

OFP, (2004). Documento propositivo en construcción. Memorias I. Movimiento social de mujeres en contra de la guerra. Barrancabermeja: Organización Femenina Popular.

POTTHAST, B. (2010). Madres, obreras, amantes... Protagonismo femenino en la historia de América Latina. Madrid: Iberoamericana.

PINEDA, R. (1997). "Mujeres entre la guerra y la paz: Lisístratas colombianas a las puertas de un nuevo milenio", Conferencia presentada en el V Congreso Nacional de la Unión de Ciudadanas de Colombia, Cali

PRIETO, P. Y TAMAYO, L. M. (2004). Las mujeres colombianas en busca de la paz: una aproximación a sus iniciativas y propuestas implementando la resolución 1325. Bogotá: Unifem.

RADCLIFFE, S. Y WESWOOD, S. (eds.). (1993). 'Viva': Woman and Popular Protest in Latin America. London: Routledge.

RAMÍREZ. P. (2009). "El movimiento de mujeres frente al conflicto armado”. En: Revista Foro (67), 31-43.

SÁNCHEZ, O. (2006). Nuevas formas de resistencia civil de lo privado a lo público: movilizaciones de la Ruta pacifica 1996-2003. Bogotá: Ruta Pacífica de la Mujeres.

. (1995). "El movimiento social de mujeres". En: Velásquez, M. (dir.) Las mujeres en la historia de Colombia (pp.379-402). Bogotá: Consejería Presidencial para la Política Social y Editorial Norma.

STEPHEN, L. (1997). Women and Social Movements in Latin America. Power From Below. Austin: University of Texas Press.

TOBÓN, G. Y MARTÍNEZ, M. (eds.) (2003). El tiempo contra las mujeres. Debates feministas para una agenda de paz. Bogotá: Humanizar.

VARGAS, V. (2008). Feminismos en América Latina. Su aporte a la política y a la democracia. Lima: Universidad Nacional Mayor de San Marcos, Centro Flora Tristán. . (2002). "Los feminismos latinoamericanos en su tránsito al nuevo milenio. (Una 
lectura político personal)”. En: Mato, D. (ed.). Estudios y otras prácticas intelectuales latinoamericanas en cultura y poder. Caracas: CLACSO. Disponible en: http://bibliotecavirtual. clacso.org.ar/ar/libros/cultura/vargas.doc Consultado: 31 agosto de 2011.

VALDÉS, T. (2000). De lo social a lo político: la acción de las mujeres latinoamericanas. Santiago de Chile: LOM Ediciones.

VELÁSQUEZ, M. (2000). "Anotaciones para una postura feminista en torno a las mujeres, la guerra y la paz”. En: Revista Nova \& Vetera (40), 103-140.

VILLARREAL, N. (2007). "Colombia: Violencias, conflicto armado y resistencias de género: las apuestas de una cartografía de la esperanza". En: Otras Miradas. 7(1), 50-66.

. (1997). "Mujeres y madres en la Ruta por la paz". En: Arango, L. G. (ed.). La crisis sociopolítica colombiana. Un análisis no coyuntural de la coyuntura (pp.363-396). Bogotá: CESUniversidad Nacional de Colombia.

. (1995). "Mujeres y espacios políticos”. En:Velásquez, M. (dir.) Las mujeres en la historia de Colombia (pp. 319-347). Bogotá: Consejería Presidencial para la Política Social y Editorial Norma.

VILLARREAL, N. Y LUNA, L. (2011). Movimientos de mujeres y participación política, Colombia del Siglo XX al XXI. Colombia: Editorial Gente Nueva.

WILLS, M. E. Y GÓMEZ, D. (2006). “Los movimientos sociales de mujeres (1970-2005). Innovaciones, estancamientos y nuevas apuestas". En: Leal, F. (ed.) En la encrucijada. Colombia en el siglo XXI (pp. 291-321). Bogotá: Norma.

WILLS, M. E. (2007). Inclusión sin representación. La irrupción política de las mujeres en Colombia 1970-2000. Bogotá: Norma. 